ISBN 978-93-84422-79-0

9th International Conference on Recent Trends in Science, Engineering, Computers and Technology

(RTSECT-2017)

Singapore Aug. 10-11, 2017

\title{
VLC Electronic Label for Hypermarket
}

\author{
Po-Cheng $\mathrm{Su}^{2}$ and Ya-Hsin Hsueh ${ }^{1 *}$ \\ 1* Department of Electronic Engineering, National Yunlin University of Science and Technology, \\ Douliu, Yunlin, Taiwan \\ Email: hsuehyh@yuntech.edu.tw \\ ${ }^{2}$ Department of Electronic Engineering, MS in Circuits and Systems, National Yunlin University of Science \\ and Technology, Douliu, Yunlin, Taiwan
}

\begin{abstract}
This work developed a multi-function electronic label of product for hypermarket which mainly aims at improving the product information and safety for both consumers and hypermarket. This device applies the techniques of visible light communication (VLC) and adopts existing LED lights from hypermarket to manage uniformly all contents of product labels and provide emergency light. Master control system on hypermarket server can control LED lights to transmit signals to the electronic label. At the same time, when electronic label on product shelves from supermarket receives the signals, the label displays the product information such as price, calories and so on. The emergency lights on the both sides of the label display board will illuminate during the blackout and lead consumers to escape from the hypermarket safely.
\end{abstract}

Keywords: LED, visible light communication (VLC)

\section{Introduction}

The previous light communication usually applies infrared communication, such as a remote controller. However, its light source is easy being influenced by environmental elements during indoor communication. Comparing the interference level that affected by environmental of visible light communication (VLC) and infrared communication, VLC is comparatively more stable than infrared communication [1] and enables to enhance data transmission speed to Mbit/s [2-6]. VLC possesses the advantages of lighting and data transmission, therefore, it has a high safety since light is straight forward and unable to pass through buildings directly to transfer data [7]. Moreover, VLC technology would not create electromagnetic wave, hence when we apply VLC to send data, the around machines are not interfered by electromagnetic wave. VLC is suitable for being applied in hospitals, airports, hypermarkets and other indoor place. The current research uses VLC in application of indoor positioning: for instance, Philips applies VLC to process indoor positioning which enables customers to search needed products [9]. Present hypermarket has no improvement for labels, thus we reform electronic label by using visible light communication.

Our VLC electronic label system enables to add product information on the label in the hypermarket. This system reduces the waste of paper resources, which achieves eco-friendly goal. In addition, there is a replenishment button on the electronic label for saving time and improving the replenishment efficiency. In emergent circumstance, the LED lights on the both sides of label illuminate and can lead consumers to escape safely.

This research was partially supported by Ministry of Science and Technology grant Most 105-2221-E-224-062. 


\section{VLC Electronic Label System}

This system structure employs two-way transmission to transmit information of product in the master control system on hypermarket server. The server applies RS232 to convey messages to VLC lighting module and transfer to special optical signal for transmission. In the following step, the VLC receiver decodes received data and displays information on the product on the label. In addition, when clicking replenishment button, the system returns a commodity information to the server directly so that the master control system enables to record out-of-stock products and replenishment immediate.

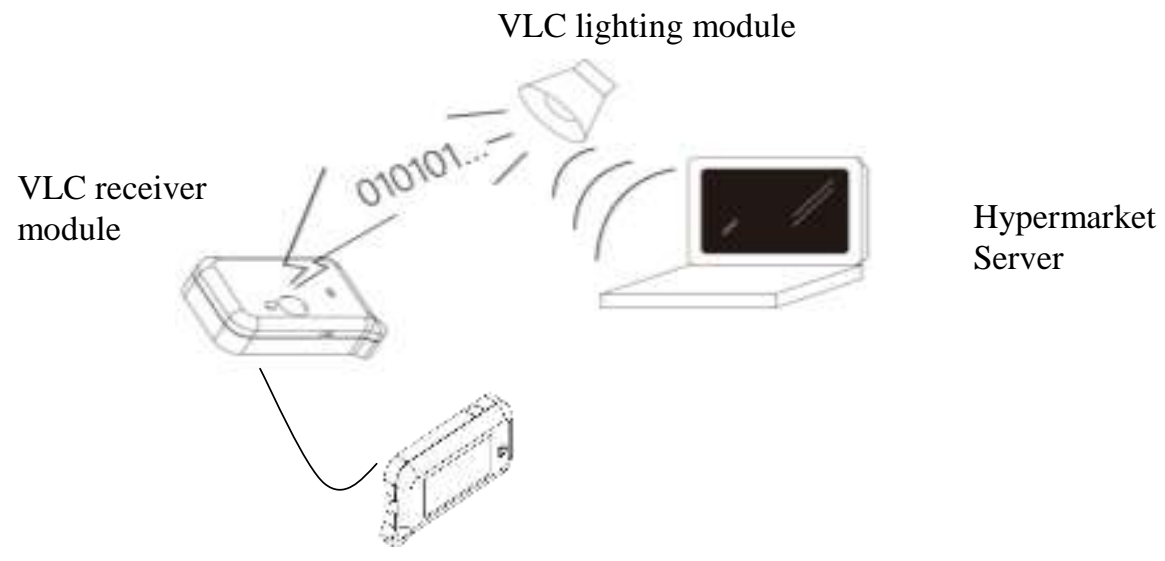

label display \& emergency light

Fig. 1.System block diagram

\subsection{Hypermarket Server}

On the server, it can control VLC lighting module to transmit product information and update them aiming at various products, as flowchart presented in Fig. 2. The baud rate is 115200 bps via connecting RS232 with VLC lighting module, the transmit protocol contains "Head", "Length", "Data", and "Checksum", as Table I shows. The data packets include "quantity", "product name", "price", "weight", "calorie", and "source area". The format of "price, "calorie", and "weight" number of two bytes; on the other hand, "product name", the "source area", and other content are transmitted as format of number, as Table II presents.

Each packet byte format of this system is 8-bit, and the first byte of content in "Head" is "V", and the second byte is "L", which confirm whether the packet is an identification code. Third byte defines that the length of the packet consists with receiving and fourth to fifteenth bytes transmit information in string or number format. After transmitting, sixteenth byte inspects the validity of information. If the above - mentioned statement is accurate, there is a complete information transmission.

TABLE I: VLC Light Control Protocol

\begin{tabular}{|c|c|c|c|c|c|}
\hline 0 & 1 & 2 & $\ldots$ & 14 & 15 \\
\hline Head & Length & Data & Checksum \\
\hline VL & 16 & $\ldots$ & \\
\hline
\end{tabular}

TABLE II: Content of Data in Packets

\begin{tabular}{|c|c|c|c|c|c|c|c|c|c|c|}
\hline & Data1 & Data2 & Data3 & Data4 & Data5 & Data7 & Data8 & Data9 & Data10 & Data11 \\
\hline content & Quantity & Price & Calorie & $\begin{array}{c}\text { Special } \\
\text { price }\end{array}$ & $\begin{array}{c}\text { Product } \\
\text { Name }\end{array}$ & $\begin{array}{c}\text { Source } \\
\text { Area }\end{array}$ & Weight \\
\hline $\begin{array}{c}\text { text } \\
\text { format }\end{array}$ & Number & Number & Number & Number & Number & Number & Number \\
\hline
\end{tabular}




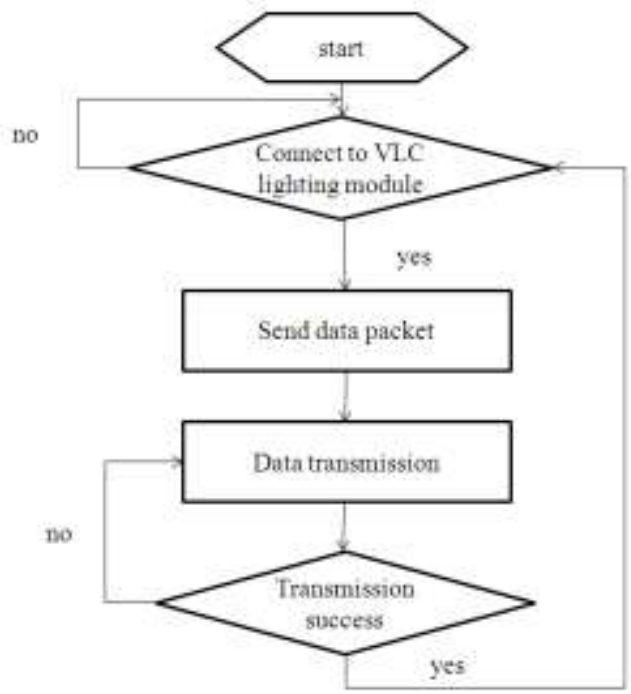

Fig. 2.Server control flow chart

\subsection{VLC Electronic Label}

First, the photodiode converts optical signals that VLC lighting transfers into an electric signal at receiving end. Second, the system amplifies signal through OP amplifier and transform it into a binary signal via threshold comparator. Third, the system analyzes the content of data packet through MCU (Cypress CY8C3866AXI) and shows the information on the LCM display.

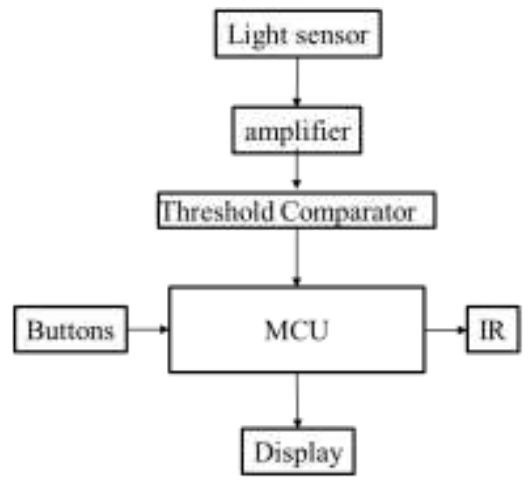

Fig.3. Electronic Label block diagram

\subsubsection{VLC Receiver module}

In the module of optical receiver, this system employs Si PIN photodiode (S6801, Hamamatsu), which generates photocurrent according to the brightness of the incident light. It transforms photocurrent into optical signal by trans-impedance amplifier and subsequently regulates voltage waveform into binary. Fig. 4 presents the structure of transforming optical receiver to an optical signal.

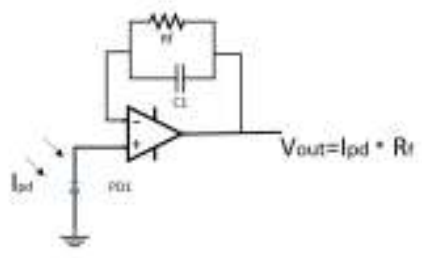

Fig.4.Circuit of optical receiver 
The work flow chart of whole electronic label system is showed in Fig. 5. First, the system estimates whether receives data packet and saves data. Second, the system judges whether clicks function button (button1) or replenishment button (button2) and presents related information on LCM display.

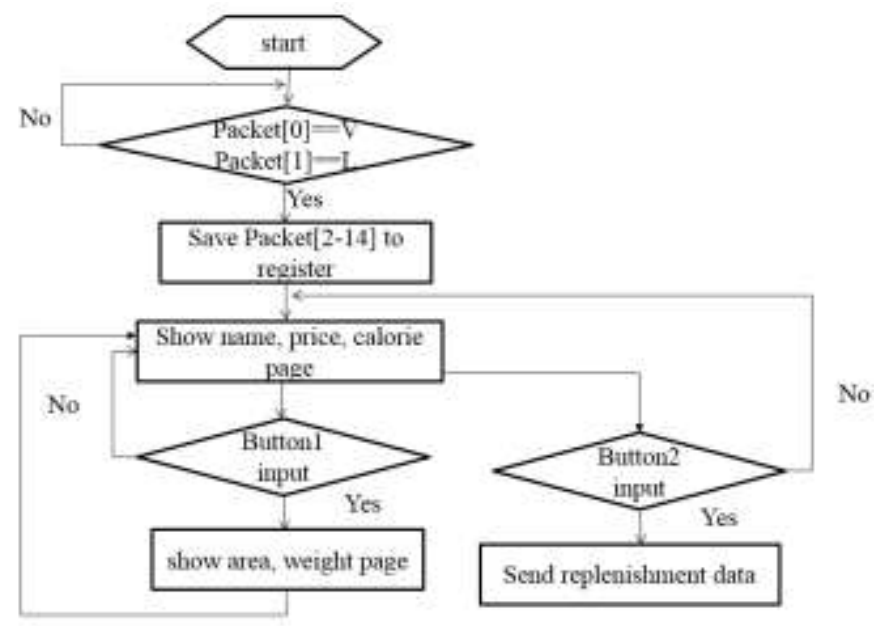

Fig.5:VLC electronic label working flow chart

\subsubsection{Other Functions}

When there are emergency circumstances like fire, earthquake, and so on which cause label being unable to supply power, the electronic label switches to supply power by built-in battery. In the meanwhile, the both sides of the electronic label illuminate which provide a clearer escape route.

When products are out of stock from shelves in hypermarket, staff can click button to convey message to the VLC lighting module (IR receiver) via IR and display the result on master control system, as showed in Fig. 6.

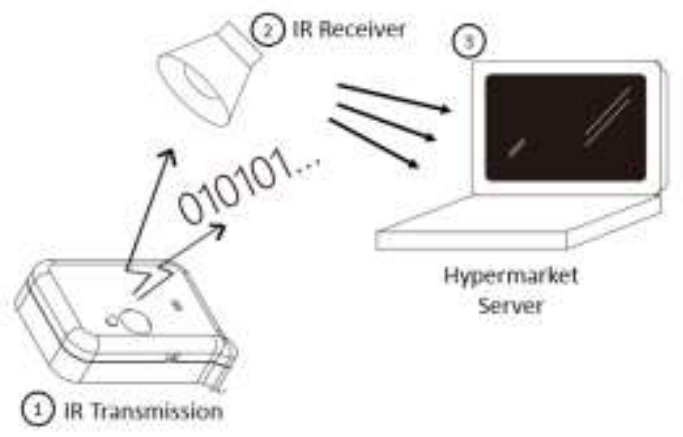

Fig. 6.System diagram

The transmission protocol of IR is the same with VLC label which the packets include "head", "length", "data", and checksum. While transmission content in head presents as "I", "R", the server saves packet data and displays the number of remaining goods on master control system GUI

\section{Implementation}

This electronic label for hypermarket has achieved product display (Fig. 7) and function of conveying replenishment message (Fig. 8). When encountering power failure, it will automatically turn on the both sides LED of electronic label and lead customers escape safely. 
The maximum characters display product name in label is up to 20 . The contents of price, weight and calorie are as the format of number and the maximum number is 255 . Moreover, source area is displayed as string, and the maximum is ten characters.

The entire system showed in Fig. 9, the distance between VLC Lighting module and VLC receiver can reach $300 \mathrm{~cm}$, and the system enables to update real-time information. We employ VLC-210GP module (Raising Technology Co. Ltd.) to implement this application.

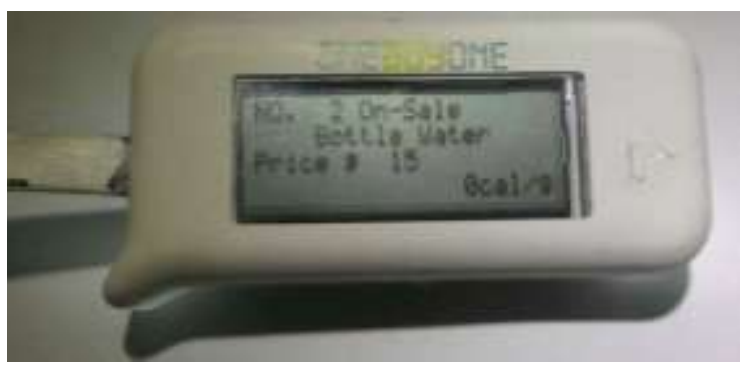

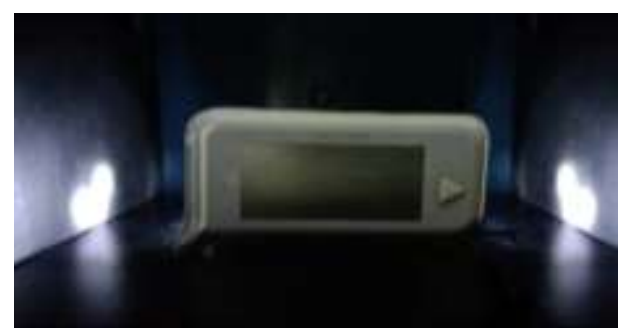

(Emergency LED when power failure)

Fig. 7. Label display

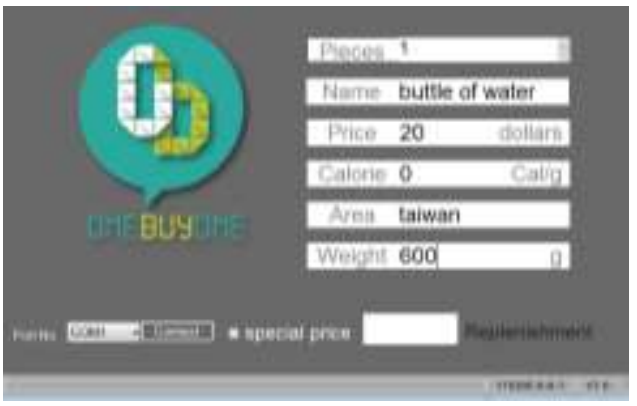

Fig. 8. Master control system GUI

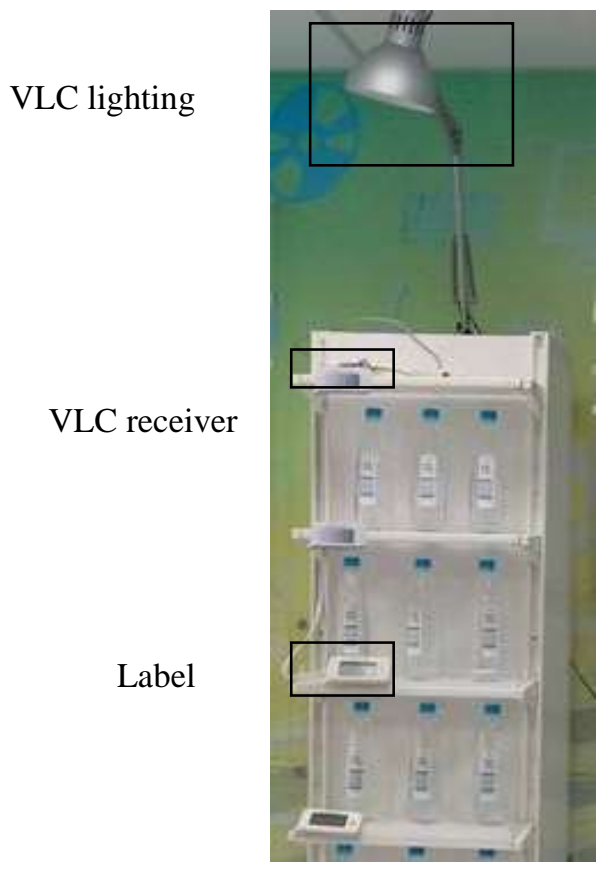

Fig. 9. Entire system 


\section{Conclusion}

Every commodity area in hypermarket has illumination lights. Therefore, the technology of VLC is not only applied in indoor positioning in hypermarket but also suitable for transmitting product information. At this work, we reform current paper label of product to electronic label that reduces the waste of paper resources in ecofriendly approach and saves human resource. Furthermore, the system increases the display of product information and provides customers more complete contents. When products are out of stock from shelves, staff replenishes products quickly and system conveys and records stock out information after clicking replenishment button which enhance efficiency.

Guiding escape route is also an issue in hypermarket. The electronic label starts emergency power after detecting power failure, the both sides LED of label display board turn on and lead customers escape safely. To sum up, this system combines multi-functions that hypermarket requires into this electronic label system to achieve.

\section{Acknowledgements}

The authors would like to thank Chiao-Yu Chang and Hung -Ling Chen for the UI interface design.

\section{References}

[1] K. Lee, H. Park, and J.R. Barry," Indoor Channel Characteristics for Visible Light Communications," IEEE Communications Letters, vol. 15,no. 2,pp.217-219, Feb. 2011.

[2] C. W. Chow, C. H. Yeh, Y. Liu,andY. F. Liu, "Digital Signal Processing for Light Emitting Diode Based Visible Light Communication," IEEE Photon. Soc. News, vol. 26, no.5, pp. 9-13, 2012

[3] H. Li, X. Chen, D. Tang, and H. Chen," High Bandwidth Visible Light Communications Based on a Post-Equalization Circuit, ”IEEE Photonics technology letters, vol. 26, no. 2, pp. 119-122, Jan.2014.

[4] Hoa Le Minh et al., "80 Mbit/s Visible Light Communications using pre-equalized white LED," in Proc. 34th European Conf. on Optical Communication, pp. 1-2, Sept. 2008

[5] H. Li, X. Chen, J. Guo and H. Chen, "A $550 \mathrm{Mbit} / \mathrm{s}$ real-time visible light communication system based on phosphorescent white light LED for practical high- speed low-complexity application," Optics Express, vol. 22, no. 22, pp. 27203-27213, 2014.

[6] H. Qian, J. Chen, S. Yao, Z. Y. Zhang, H. Zhang and W. Xu, "One-Bit Sigma-Delta Modulator for Nonlinear Visible Light Communication Systems," IEEE Photonics Technology Letters, vol. 27, no. 4, pp. 419-422, Feb. 2015.

[7] M. Moon and S. Choi, "Indoor Position Estimation Using Image Sensor Based on VLC", The 2014 International Conference on Advanced Technologies for Communications , 2014

[8] Gordonpovey, 2011, “Top 10 Visible Light Communications Applications”, WordPress. [Online] http://visiblelightcomm.com/top-10-visible-light-communications-applications/

[9] Philips indoor positioning: the game changer in retail,

[Online] http://www.lighting.philips.com/main/systems/themes/led-based-indoor-positioning 